\section{Physical activity does not influence risk of developing knee OA}

Middle-aged and elderly individuals are encouraged to exercise regularly, but some reports have raised concern that exercise might worsen the risk of developing knee osteoarthritis $(\mathrm{OA})$ in overweight individuals. Previous studies in this area produced discordant results, so Felson and colleagues evaluated the long-term effect of recreational exercise on development of knee $\mathrm{OA}$ in a well-characterized, community-based group of adults (the Framingham Offspring cohort), many of whom were overweight.

The study included 1,279 individuals (mean age at baseline 53.2 years) without evidence of $\mathrm{OA}$, who completed a questionnaire on exercise and activity levels, underwent anteroposterior and lateral radiography of both knees, and answered questions about knee symptoms at baseline and after $\sim 9$ years. Recreational exercise-walking, jogging, working up a sweat, and high activity levels relative to those of peers-did not increase or decrease participants' risk of developing knee $\mathrm{OA}$, even for individuals who were overweight or obese (mean BMI $31.1 \pm 3.4 \mathrm{~kg} / \mathrm{m}^{2}$ in men and $30.7 \pm 5.0 \mathrm{~kg} / \mathrm{m}^{2}$ in women). Overweight individuals had an increased risk of developing knee OA, but their risk was unaffected by exercise. Joint-space narrowing was also unaffected by participants' exercise levels.

These findings suggest that exercise does not protect middle-aged or older individuals against the development of knee OA but, on the other hand, physical exercise can be done safely even by overweight persons, without concern that they will consequently develop knee OA.

Original article Felson DT et al. (2007) Effect of recreational physical activities on the development of knee osteoarthritis in older adults of different weights: the Framingham study. Arthritis Rheum 57: 6-12

\section{Intravenous pulsed cyclophosphamide benefits patients with systemic sclerosis}

Interstitial lung disease is the most common cause of death in patients with systemic sclerosis. Cyclophosphamide, with or without steroids, is thought to modify the course of lung disease in these patients, but this treatment is considered empirical. Yiannopoulos and colleagues evaluated the efficacy and safety of intravenous pulsed cyclophosphamide and methylprednisolone in the treatment of systemic sclerosis lung disease.

Their prospective study involved 13 patients (mean age 54.5 years, 9 women) with systemic sclerosis and lung involvement. Participants underwent high-resolution CT of the lungs at baseline and after 24 months, and their forced vital lung capacity and carbon monoxide diffusion lung capacity were assessed at baseline and after 6, 12, 24 and 48 months. Treatment comprised six once-monthly pulses of 750 $1,000 \mathrm{mg} / \mathrm{m}^{2}$ intravenous cyclophosphamide plus $1 \mathrm{~g}$ methylprednisolone, followed by 12-18 months of bimonthly pulses. Between infusions, patients received 6-8 mg oral methylprednisolone daily. There were no serious adverse effects of treatment.

CT findings correlated poorly with lung function test results. Lung function mildly improved or stabilized after 24 months in $76.6 \%$ of patients-most of whom had initially nonsevere functional impairment-but this proportion fell to $58.3 \%$ after treatment ceased; the authors speculate that maintenance courses of this or other immunosuppressive treatments might be required. They emphasize the need for early use of aggressive immunosuppressive therapy in patients with systemic sclerosis, before severe functional lung impairment develops.

Original article Yiannopoulos G et al. (2007) Combination of intravenous pulses of cyclophosphamide and methylprednizolone in patients with systemic sclerosis and interstitial lung disease. Rhumatol Int 27:357-361

\section{High prevalence of metabolic syndrome in patients with gout or SLE}

The metabolic syndrome comprises multiple conditions, and is an independent risk factor for cardiovascular morbidity and type II diabetes. Studies have indicated an increased prevalence of metabolic syndrome in patients with gout or systemic lupus erythematosus (SLE), and two independent quantitative studies have now confirmed these findings.

The first study included 102 patients with SLE and 101 controls. The metabolic syndromedefined according to National Cholesterol Education Program Adult Treatment Panel III 